\title{
Erratum to: Elementary Mathematics
}

\section{Erratum to:}

\section{Chapter 1, in: P. Olsson, Transport Phenomena}

in Newtonian Fluids - A Concise Primer, SpringerBriefs in Continuum Mechanics, DOI 10.1007/978-3-319-01309-1_1

There is a change in Eq. (1.8). The term " 0 " is deleted and equation should read as below:

$$
\begin{aligned}
\nabla \times \mathbf{F} & =\left|\begin{array}{ccc}
\widehat{\mathbf{x}}_{1} & \widehat{\mathbf{x}}_{2} & \widehat{\mathbf{x}}_{3} \\
\frac{\partial}{\partial x_{1}} & \frac{\partial}{\partial x_{2}} & \frac{\partial}{\partial x_{3}} \\
F_{1} & F_{2} & F_{3}
\end{array}\right| \\
& =\widehat{\mathbf{x}}_{1}\left(\frac{\partial F_{3}}{\partial x_{2}}-\frac{\partial F_{2}}{\partial x_{3}}\right)+\widehat{\mathbf{x}}_{2}\left(\frac{\partial F_{1}}{\partial x_{3}}-\frac{\partial F_{3}}{\partial x_{1}}\right)+\widehat{\mathbf{x}}_{3}\left(\frac{\partial F_{2}}{\partial x_{1}}-\frac{\partial F_{1}}{\partial x_{2}}\right)
\end{aligned}
$$

The online version of the original chapter can be found under DOI 10.1007/978-3-319-01309-1_1

P. Olsson ( $ه)$

Arvid Lindmansgatan 2A, 41726 Göteborg, Sweden

e-mail: po.systemanalys@telia.com

P. Olsson, Transport Phenomena in Newtonian Fluids - A Concise Primer,

E1 SpringerBriefs in Continuum Mechanics,

DOI: 10.1007/978-3-319-01309-1_5, (C) The Author(s) 2014 